\title{
Avaliação biomecânica de atletas paraolímpicos brasileiros
}

\author{
Sílvio Soares dos Santos $^{1}$ e Fernando José de Sá Pereira Guimarães ${ }^{2}$
}

\section{RESUMO}

A biomecânica do esporte é uma disciplina científica da qual os movimentos desportivos são descritos e explicados à luz de conceitos e métodos mecânicos ${ }^{1}$. De acordo com a área de aplicação a biomecânica pode ser subdividida em biomecânica do rendimento, biomecânica antropométrica e biomecânica preventiva. A biomecânica do rendimento está diretamente relacionada à análise da técnica do movimento, cujas funções são a identificação e avaliação das variáveis de influência e o diagnóstico individual em relação às falhas técnico-motoras registradas no movimento ${ }^{2}$. A biomecânica utiliza como métodos de medição de seus parâmetros quantitativos a cinemetria, a eletromiografia, a dinamometria e a antropometria ${ }^{3,4}$. O objetivo desse trabalho foi fazer análises quantitativa e qualitativa de parâmetros biomecânicos de provas de atletismo e natação, utilizando a cinemetria. Os resultados mostraram imperfeições na condução da técnica dos movimentos, em especial, das disciplinas de arremessos no atletismo e na natação. Tais resultados serviram como subsídios para os treinadores adaptarem e modificarem seus treinamentos no sentido de corrigir tais imperfeições.

Palavras-chave: Biomecânica. Esporte paraolímpico. Cinemática. Análise qualitativa. Análise quantitativa.

\section{ABSTRACT}

\section{Biomechanic evaluation of Brazilian paralympic athletes}

Sports biomechanics is a scientific discipline that uses mechanical methods and concepts to study sports-related

1. Professor Adjunto II - Faculdade de Educação Física, Universidade Federal de Uberlândia (UFU).

2. Professor da Universidade de Pernambuco (UFPE).

Submetido em: 4/4/02

Versão revisada recebida em: 1/5/02

Aceito em: 19/5/02

Endereço para correspondência:

Rua Benjamin Constant, 1.286

38400-678 - Uberlândia, MG

E-mail: silvio@ufu.br movements and can be divided into performance biomechanics, anthropometric biomechanics, and preventive biomechanics ${ }^{4}$. Performance biomechanics is directly related to the analysis of the technique of the movements in order to identify and evaluate the main variables involved, and to allow individual diagnosis related to important flaws (technique versus motor control) registered in the movement ${ }^{2}$. According to Winter ${ }^{3}$ and Baumann ${ }^{4}$, the usual methods to measure biomechanic quantitative parameters are based on kinemetry, electromyography, dynamometry and the anthropometrics. The aim of this work was to perform quantitative and qualitative evaluations of biomechanic parameters related to swimming and athletics, based on kinemetry. The results showed a number of imperfections for correctly executing the technique related to a movement (this was most pronounced for throwing and swimming). These data can now be used by coaches looking for new means of adjusting their techniques in order to correct possible imperfections.

Key words: Biomechanics. Paralympic sports. Kinemetry. Qualitative analysis. Quantitative analysis.

\section{INTRODUÇÃO}

A biomecânica do esporte é uma disciplina científica da qual os movimentos desportivos são descritos e explicados à luz de conceitos e métodos mecânicos. De acordo com a área de aplicação a biomecânica pode ser subdividida em: biomecânica do rendimento - se ocupa das variáveis biomecânicas que determinam o resultado do movimento em qualquer nível de rendimento; biomecânica antropométrica - se relaciona com o diagnóstico e prognóstico do rendimento relativamente às medidas antropométricas e biomecânica preventiva - se relaciona com a identificação de cargas e os possíveis desgastes ocasionados ao aparelho de movimento oriundos da aplicação dessas cargas.

A biomecânica do rendimento está diretamente relacionada à análise da técnica do movimento, cujas funções são a identificação de suas variáveis de influência, a avaliação dessas variáveis e por fim o diagnóstico individual em relação às falhas técnico-motoras registradas no movimento $^{2}$. 
A biomecânica utiliza como métodos de medição de seus parâmetros quantitativos a cinemetria, a eletromiografia, a dinamometria e a antropometria ${ }^{3,4}$.

A cinemetria é um método de medição cinemática que busca a partir da aquisição de imagens da execução do movimento, observar o comportamento de variáveis dependentes, tais como: velocidade, deslocamento, posição e orientação do corpo e de suas partes ${ }^{5}$.

A dinamometria engloba todos os tipos de medidas de força de forma a poder interpretar as respostas de comportamentos dinâmicos do movimento humano.

A eletromiografia é o método que registra a atividade elétrica do músculo quando esse realiza contração.

A antropometria procura determinar características e propriedades do aparelho locomotor como as dimensões das formas geométricas de segmentos corporais, distribuição de massa, braços de alavanca, posições articulares etc., definindo um modelo antropométrico a partir das medidas corporais ${ }^{5}$.

\section{METODOLOGIA}

Para a avaliação biomecânica dos atletas de corrida no atletismo foi utilizado o método cinemétrico. Os atletas foram filmados durante a execução de suas provas específicas e avaliados os seguintes parâmetros: velocidade média parcial, velocidade média total, comprimento médio da passada e freqüência média da passada. As filmagens foram feitas perpendicularmente ao desenvolvimento das corridas. A filmadora foi fixada no ponto médio da corrida e colocados pontos de referência ao fundo, eqüidistantes entre si, cujas distancias eram conhecidas. Os testes foram realizados na cidade de São Paulo numa pista sintética oficial.

Foi desenvolvida uma lista de checagem para a análise qualitativa de cada uma das diferentes provas de atletismo, divididas em provas de corridas e de arremessos. As provas de arremessos foram ainda subdivididas em arremessos de peso, de disco e de dardo. Foram utilizadas duas filmadoras para a captura de imagens dos arremessos. Essas filmadoras foram colocadas à frente dos arremessadores (aproximadamente 15m) e com 90 graus de afastamento entre si.

Na disciplina natação foram feitas filmagens subaquáticas nos planos transverso e sagital em uma piscina de $25 \mathrm{~m}$ $\mathrm{x} 12,5 \mathrm{~m}$. Foram analisadas as saídas, as viradas e a técnica de nado desenvolvida. Foram feitas ainda filmagens fora da água para a análise das saídas (fase aérea) e a técnica de nado.

A filmadora foi fixada abaixo do nível da água (aproximadamente $20 \mathrm{~cm}$ de profundidade) a $1,5 \mathrm{~m}$ da borda de fundo da piscina para a observação das viradas. Para a observação das saídas a filmadora foi fixada a aproximadamente $3 \mathrm{~m}$ da borda de fundo. Para as filmagens dos nados no plano sagital a filmadora foi fixada a $12,5 \mathrm{~m}$ da borda de fundo e distante dos nadadores cerca de $8 \mathrm{~m}$. As filmagens no plano transverso foram feitas com a filmadora colocada na posição $25 \mathrm{~m}$ na mesma direção de desenvolvimento do nado. Puderam ser observados cerca de 6 ciclos completos de braçada para cada nadador nos vários planos.

\section{RESULTADOS}

Os resultados das análises biomecânicas serão apresentados na seguinte ordem: 1) atletismo - corridas; 2) atletismo - arremessos e 3) natação.

\section{1) Atletismo}

Dentro dessa disciplina foram analisadas principalmente as provas curtas de pista (corridas de $100 \mathrm{~m}, 200 \mathrm{~m}$ e $400 \mathrm{~m}$ ) e as provas longas de $10000 \mathrm{~m}$ e maratona.

Dentro das provas de campo foram analisadas as provas de arremessos de peso, dardo e disco.

\section{- Corridas}

A tabela 1 mostra os resultados dos atletas para o teste de 100 metros rasos. São descritas as velocidades médias $(\mathrm{m} / \mathrm{s})$ a cada 25 metros.

\begin{tabular}{lccccc}
\hline \multicolumn{6}{c}{ TABELA 1 } \\
\multicolumn{5}{c}{$\begin{array}{l}\text { Velocidades parciais dos atletas para } \\
\text { o teste de 100 } \\
\text { metros rasos em } \mathbf{~ m / s ~}\end{array}$} \\
\hline & V(0-25) & V(25-50) & V(50-75) & V(75-100) & Vmedia \\
A.S. & 7,06 & 7,14 & 8,71 & 8,39 & 7,76 \\
M.J. & 6,41 & 7,35 & 8,22 & 7,58 & 7,33 \\
A.L. & 7,14 & 9,16 & 10,96 & 8,65 & 8,77 \\
A.D. & 7,35 & 8,99 & 9,62 & 9,54 & 8,77 \\
S. & 6,98 & 8,45 & 10,59 & 7,91 & 8,29 \\
\hline
\end{tabular}

A figura 1 mostra os resultados das atletas da tabela 1 em forma de gráfico de coluna. As atletas avaliadas mostram essencialmente a mesma estrutura. A atleta M.J. mostra de maneira mais bem definida um aumento progressivo da velocidade até os 75 metros. Ambas as atletas mostraram uma queda proporcional nos últimos 25 metros. As seqüências de 1 a 4 na figura abaixo representam as distancias de $0-25 \mathrm{~m}, 25-50 \mathrm{~m}, 50-75 \mathrm{~m}$ e $75-100 \mathrm{~m}$ respectivamente. 


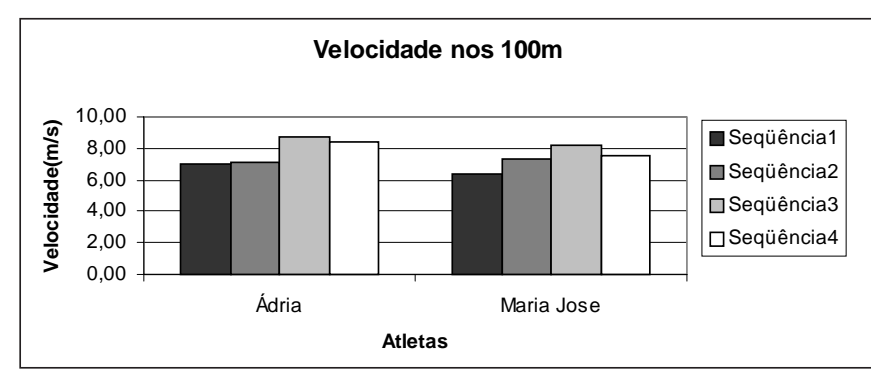

Fig. 1 - Gráfico de velocidades parciais no teste de $100 \mathrm{~m}$ rasos das atletas de atletismo

A figura 2 mostra os resultados dos atletas na prova de 100 metros rasos cujos resultados encontram-se na tabela 1. A estrutura desses gráficos é basicamente a mesma inclusive em comparação à figura das atletas mostradas na figura 1, ou seja, aumento progressivo da velocidade até os 75 metros e uma queda da mesma entre os 75 e 100 metros. O atleta A.D. mostra um gráfico um pouco diferenciado com pequena queda da velocidade entre os 75 e 100 metros. Observando-se a figura 2 pode-se ver que, aparentemente, o atleta A. mostra melhor performance que seu colega D., devido aos altos picos de velocidade atingidos pelo primeiro. No entanto, a observação da tabela 1 mostra que ambos apresentaram a mesma velocidade média. Talvez seja uma boa estratégia controlar a velocidade durante toda a prova no sentido de não sofrer uma grande queda na mesma no último quarto da prova e distribuindo melhor, dessa forma, o gasto energético durante a mesma.

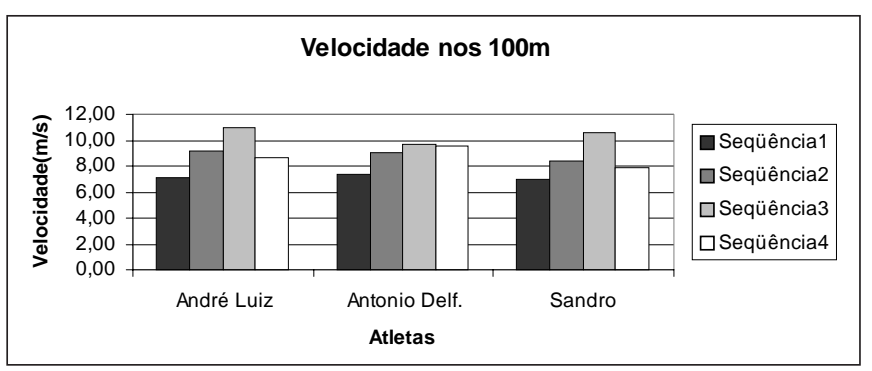

Fig. 2 - Gráfico de velocidades parciais no teste de $100 \mathrm{~m}$ rasos dos atletas de atletismo

A tabela 2 mostra a velocidade média apresentada pelos atletas assim como a frequiência média de passada e o comprimento médio da passada no teste dos $100 \mathrm{~m}$ rasos. Destaca-se nessa tabela o comprimento médio de passada do atleta $\mathrm{S}$. $(4,76 \mathrm{~m} /$ ciclo), que é bastante alto, em contrapartida a frequiência de suas passadas $(1,74$ ciclos/seg) que é extremamente baixo. $\mathrm{O}$ aumento de $5 \%$ na frequiência das passadas no atleta $\mathrm{S}$. com a manutenção do seu comprimento provocaria uma redução do tempo de percurso na prova de 12,06 seg para 11,49 seg.

\begin{tabular}{lccc}
\hline \multicolumn{3}{c}{$\begin{array}{c}\text { TABELA 2 } \\
\text { Velocidade média, freqüência e comprimento } \\
\text { de passadas dos atletas no teste de 100m rasos }\end{array}$} \\
\hline & $\begin{array}{c}\text { Vmédia } \\
\text { 100m (m/s) }\end{array}$ & $\begin{array}{c}\text { Freq. média } \\
\text { (ciclos/s) }\end{array}$ & $\begin{array}{c}\text { Comp. médio } \\
\text { (m/ciclo) }\end{array}$ \\
& & & \\
A.S. & 7,76 & 2,06 & 3,77 \\
M.J. & 7,33 & 1,98 & 3,70 \\
A.L. & 8,77 & 2,15 & 4,08 \\
A.D. & 8,77 & 2,15 & 4,08 \\
S. & 8,29 & 1,74 & 4,76 \\
\hline
\end{tabular}

A tabela 3 mostra os parâmetros velocidade média, freqüência e comprimento médio de passadas dos atletas no teste de $400 \mathrm{~m}$ rasos. Em relação à atleta A.S. pode-se notar que houve uma manutenção do comprimento de passadas porém a redução da velocidade, em relação ao teste dos $100 \mathrm{~m}$ rasos, deveu-se principalmente à redução da freqüência média das passadas durante esse segundo teste. O mesmo fenômeno pode ser observado com os atletas A. D. e S.

\begin{tabular}{|c|c|c|c|}
\hline \multicolumn{4}{|c|}{$\begin{array}{c}\text { TABELA } 3 \\
\text { Velocidade média, comprimento e freqüência } \\
\text { média das passadas no teste de } 400 \mathrm{~m} \text { rasos }\end{array}$} \\
\hline & $\begin{array}{c}\text { Vmédia } \\
400 \mathrm{~m}(\mathrm{~m} / \mathrm{s})\end{array}$ & $\begin{array}{l}\text { Freq. média } \\
\text { (ciclos/s) }\end{array}$ & $\begin{array}{l}\text { Comp. médio } \\
\text { (m/ciclo) }\end{array}$ \\
\hline A.S. & 6,34 & 1,68 & 3,77 \\
\hline A.D. & 7,27 & 1,79 & 4,06 \\
\hline S. & 7,47 & 1,57 & 4,76 \\
\hline
\end{tabular}

A figura 3 mostra o comportamento da velocidade dos atletas a cada 100 metros no teste dos 400 metros rasos. O atleta S. mostra uma alta velocidade nos primeiros 200 metros experimentando nos 200 metros finais uma substancial redução na mesma. Talvez seja uma estratégia interessante dosar um pouco melhor a velocidade durante toda a prova no sentido de economizar energia para o final da

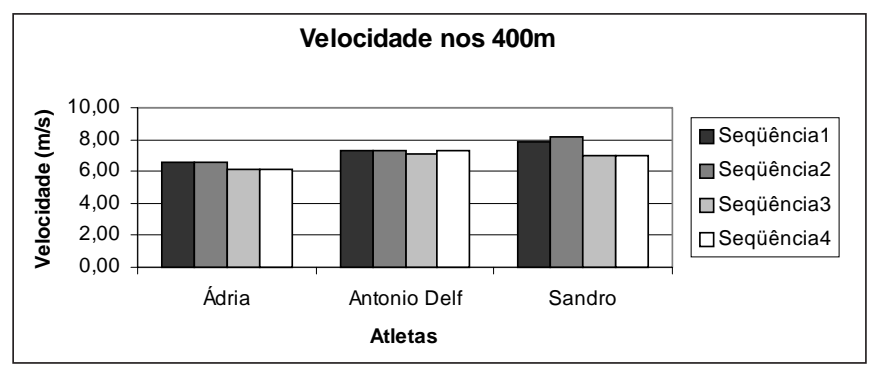

Fig. 3 - Velocidade parciais nos 400 metros rasos 
mesma. Com essa estratégia seria possível manter uma melhor velocidade média durante toda a prova. Além do aspecto energético cabe aqui a menção de outro, qual seja, a manutenção da concentração durante toda a prova. $\mathrm{O}$ mesmo fenômeno ocorreu com a A.S. porém em níveis bem menores. $\mathrm{O}$ atleta A.D. mostrou um aumento da velocidade durante as duas primeiras partes da prova, uma redução na terceira e um discreto aumento na sua parte final.

Os resultados do teste de 200 metros rasos aparecem na tabela 4 e na figura 4 . As velocidades parciais da atleta M.J. mostram um aumento até os 100 metros ocorrendo uma leve queda entre os 100 e 150 metros e uma estabilização nos 50 metros restantes.

A.L. mostrou um aumento progressivo no valor das velocidades durante toda a prova.

TABELA 4

Velocidades parciais no teste dos $200 \mathrm{~m}$ rasos em m/s

\begin{tabular}{lcccc}
\hline & $\mathbf{V}(\mathbf{0}-\mathbf{5 0})$ & $\mathrm{V}(\mathbf{5 0 - 1 0 0 )}$ & $\mathrm{V}(\mathbf{1 0 0 - 1 5 0 )}$ & $\mathrm{V}(\mathbf{1 5 0 - 2 0 0 )}$ \\
M.J. & 6,96 & 7,74 & 7,49 & 7,50 \\
A.L. & 7,81 & 9,03 & 9,28 & 9,45 \\
\hline
\end{tabular}

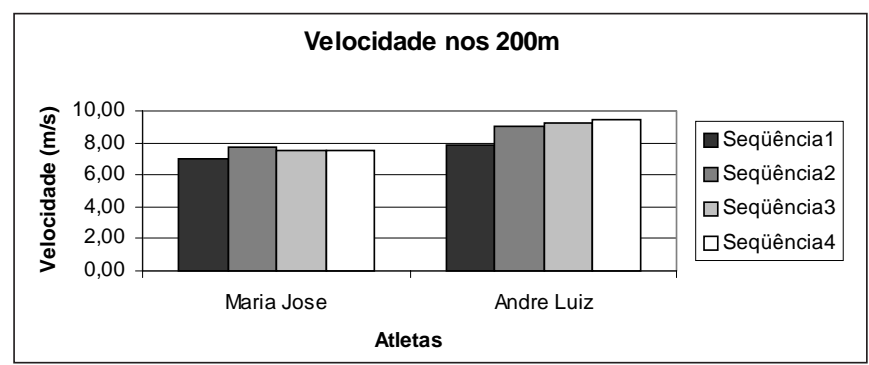

Fig. 4 - Velocidades parciais nos 200 metros rasos

A tabela 5 mostra os valores obtidos para a velocidade média, comprimento e freqüência de passadas no teste de 200 metros rasos. A frequiência média das passadas foi aumentada em relação à prova dos $100 \mathrm{~m}$ em cerca de $4 \%$, ocorrendo também uma redução de quase $4 \%$ no comprimento das passadas para a atleta M.J. O atleta A.L. mos-

TABELA 5

Velocidade média, freqüência e comprimento médio das passadas nos $200 \mathrm{~m}$ rasos

\begin{tabular}{lccc}
\hline & $\begin{array}{c}\text { Vmédia } \\
(\mathbf{m} / \mathbf{s})\end{array}$ & $\begin{array}{c}\text { Freq. média } \\
\text { (ciclo/s) }\end{array}$ & $\begin{array}{c}\text { Comp. médio } \\
(\mathbf{m} / \text { ciclo) }\end{array}$ \\
M.J. & 7,39 & 2,07 & 3,57 \\
A.L. & 8,84 & 2,06 & 4,30 \\
\hline
\end{tabular}

trou uma redução no comprimento das passadas de aproximadamente $4 \%$ e um aumento no seu comprimento em torno de $5 \%$ quando comparado com a prova dos 100 rasos.

A tabela 6 mostra as velocidades parciais a cada $400 \mathrm{~m}$ do atleta corredor de fundo, no teste dos 1600 metros. O atleta demonstra uma grande capacidade de manutenção da sua velocidade com a mesma oscilando entre seus limites superior e inferior em apenas $2 \%$.

\section{TABELA 6}

Velocidades parciais a cada $400 \mathrm{~m}$ no teste de 1.600 metros

\begin{tabular}{ccccc}
\hline & $\begin{array}{c}\mathrm{V}(\mathbf{0}-\mathbf{4 0 0}) \\
\mathbf{m} / \mathbf{s}\end{array}$ & $\begin{array}{c}\mathrm{V}(\mathbf{4 0 0}-\mathbf{8 0 0}) \\
\mathbf{m} / \mathbf{s}\end{array}$ & $\begin{array}{c}\mathrm{V}(\mathbf{8 0 0}-\mathbf{1 . 2 0 0} \\
\mathbf{m} / \mathbf{s}\end{array}$ & $\begin{array}{c}\mathrm{V}(\mathbf{1 . 2 0 0 - 1 . 6 0 0}) \\
\mathbf{m} / \mathbf{s}\end{array}$ \\
$\mathrm{A}$ & 5,35 & 5,23 & 5,24 & 5,33 \\
\hline
\end{tabular}

TABELA 7

Valores de velocidade média, freqüência e comprimento de passadas no teste de $1.600 \mathrm{~m}$

\begin{tabular}{cccc}
\hline & $\begin{array}{c}\text { Vmédia } \\
(\mathbf{m} / \mathbf{s})\end{array}$ & $\begin{array}{c}\text { Freq. média } \\
(\text { ciclo/s) }\end{array}$ & $\begin{array}{c}\text { Comp. médio } \\
(\mathbf{m} / \text { ciclo) }\end{array}$ \\
$\mathrm{A}$ & 5,29 & 1,43 & 3,70 \\
\hline
\end{tabular}

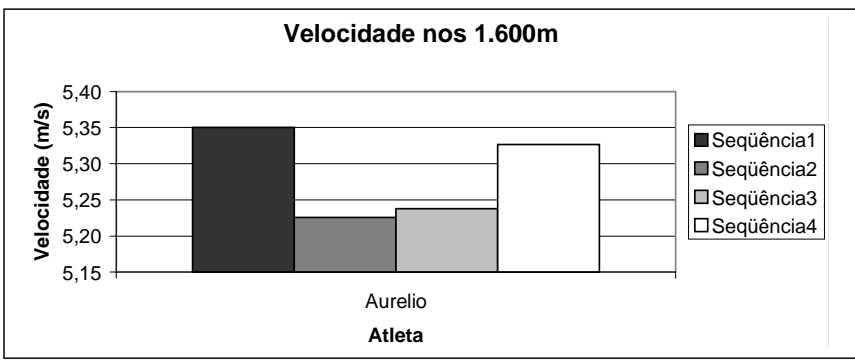

Fig. 5 - Velocidades parciais a cada 400 m no teste de 1.600 metros do atleta fundista paraolímpico $\mathrm{A}$

Os níveis de velocidade alcançados no teste de 1.600 metros mostrados na tabela 7 , mostram a possibilidade de se correr a maratona, caso seja mantida a velocidade média de $5,29 \mathrm{~m} / \mathrm{s}$, em aproximadamente 2 horas e $20 \mathrm{minu}$ tos.

Foi observado na atleta A.S. um problema de caráter técnico que pode ter sua origem em uma lesão que a atleta apresentou há anos atrás no joelho esquerdo. Ao compararmos o padrão de flexão do joelho direito (fig. 6 seqüência B) com o joelho esquerdo para a mesma fase do movimento (fig. 6 seqüências D e E), fica notório a diferença entre ambos no aspecto da quantidade de flexão observada 


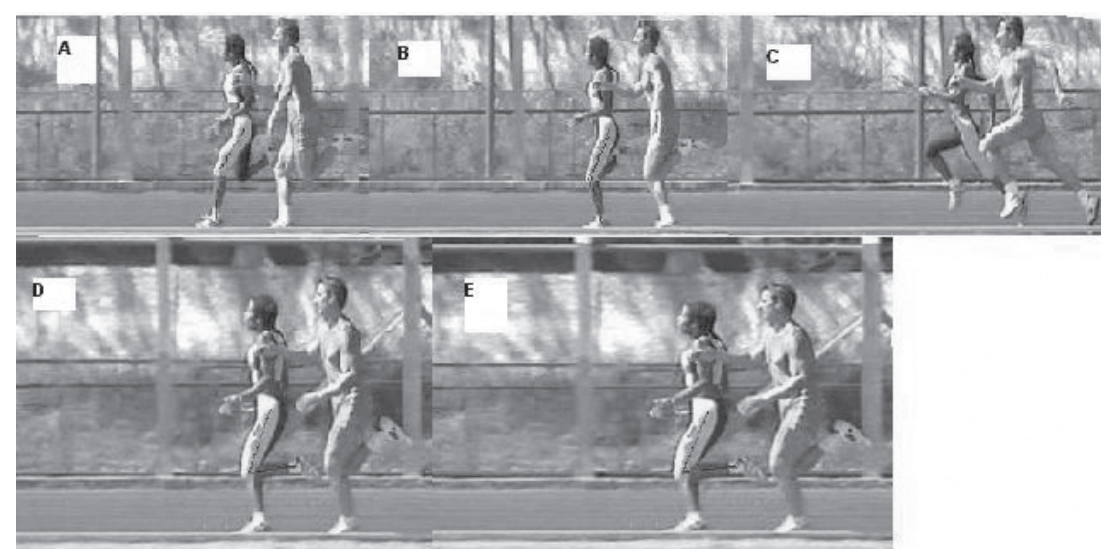

Fig. 6 - Seqüência de passadas da atleta A.S.

para a mesma fase de movimento. Enquanto na seqüência $\mathrm{B}$ o calcanhar toca nas nádegas, que é o padrão desejável para corridas de velocidade, nas sequiências D e E o joelho flexiona-se pouco mais que $90^{\circ}$ não permitindo desta forma que o calcanhar toque as nádegas. Com isso a inércia desse membro aumenta, acarretando uma sobrecarga para a musculatura impulsora do balanceamento da coxa (flexores dos quadris), além de alterar o ritmo das passadas.

Esse padrão de movimento foi observado em todas as três corridas $(100 \mathrm{~m}, 200 \mathrm{~m}$ e $400 \mathrm{~m})$. Durante a corrida de 100 metros pode ser detectado através da filmagem da prova, no plano frontal, uma ligeira inclinação da atleta para o seu lado esquerdo $\left(\mathrm{H}^{\prime \prime} 6^{\circ}\right)$, o que provoca ao longo da corrida, um desvio lateral em relação à sua trajetória ideal, aumentando dessa forma o espaço percorrido pela atleta e conseqüentemente um incremento do tempo total da corrida.

\section{2) Arremessos}

Os arremessos serão analisados de maneira genérica sob o ponto de vista da técnica e de acordo com o implemento lançado. Serão estudados os arremessos de peso (3 atletas), dardo (2 atletas) e disco (2 atletas). Os testes foram realizados na Universidade Estadual de Pernambuco na cidade do Recife.

Arremessos de peso. Foram executados por três atletas do sexo feminino que apresentavam amputação de um dos membros inferiores, uma com dupla amputação e uma terceira com AVC (acidente vascular cerebral).

A atleta que apresentava dupla amputação dos membros inferiores apresentou uma boa técnica de arremesso no entanto com alguma deficiência na fase de preparação com pouca rotação e flexão lateral da coluna. $\mathrm{Na}$ fase principal do exercício apresentou ainda um rebaixamento do cotovelo do braço de arremesso que prejudicava a potência final imprimida ao aparelho.
A atleta com amputação de um dos membros apresenta uma boa técnica de arremesso podendo no entanto ampliar sua rotação e flexão lateral do tronco.

A terceira atleta apresenta alguns problemas coordenativos, devido sobretudo à sua deficiência, mas que podem ser minimizados com alguns procedimentos. Foi sugerido a ela que intensificasse o trabalho de flexibilidade, sobretudo do tronco.

Arremesso de dardo. Foi executado pela atleta com amputação de um dos membros e aquela com AVC.

A primeira apresentou uma boa técnica carecendo no entanto de uma melhor preparação do ponto de vista físico (melhoria da amplitude dos movimentos do tronco).

Foi sugerido a essa atleta que começasse a treinar esse tipo de prova sem o auxilio da cadeira de apoio. Dessa forma, com a introdução do deslocamento horizontal ao movimento as chances de uma melhor performance seriam enormemente ampliadas, devido à possibilidade de aplicação do princípio da transmissão do impulso a esse movimento. Os problemas de desequilíbrio gerados pela utilização de apenas uma das pernas poderá ser perfeitamente minimizado e superado ao longo do período de treinamento e adaptação à essa nova técnica.

A segunda atleta apresentou os mesmos problemas nessa modalidade que aqueles apresentados no arremesso de peso. Foi sugerido a ela que fizesse um treinamento de arremesso estático, o que demandaria menor processamento de informação e provavelmente um melhor nível da performance. $\mathrm{O}$ arremesso com deslocamento apresentou uma fase de descoordenação entre as passadas de aproximação, a preparação, o bloqueio e o arremesso propriamente dito, o que prejudicou bastante a performance do movimento. Foi tentado então uma execução sem deslocamento. Executando o exercício sem deslocamento foi possível observar-se uma melhoria da fase principal devido a uma me- 
lhor preparação da fase precedente, o que não pode ser observado na execução com deslocamento.

Arremesso de disco. Foi executado pelas duas atletas com amputação dos membros inferiores e pelo único atleta arremessador.

A atleta com dupla amputação apresentou os mesmos problemas observados nos arremessos anteriores, ou seja, pouca amplitude dos movimentos no tronco, devido principalmente ao excessivo acúmulo de tecido adiposo nessa região, verificável na tabela 5 .

A outra atleta mostrou uma técnica de arremesso muito boa. Sua principal limitação encontra-se no fato de a mesma arremessar apoiada sobre uma cadeira, o que lhe impede de movimentar-se linearmente. Foi sugerido a ela que tentasse adaptar uma técnica de arremesso em movimento com o intuito de maximizar a sua performance.

$\mathrm{O}$ atleta apresenta boa técnica de arremesso, sem grandes problemas do ponto de vista técnico. Ele apresenta alguma inconstância durante os arremessos, que pode significar:

a) que o padrão de movimentos ainda não se encontra bem fixado ou,

b) baixos níveis de concentração durante os arremessos.

Deve-se ressaltar aqui que as condições encontradas para se executar todos os arremessos não eram ideais, comprometendo dessa forma, a performance dos atletas.

\section{3) Natação}

Na natação os atletas foram avaliados, na medida do possível, dentro das modalidades que iriam disputar. Os testes foram realizados na piscina da Universidade Estadual de Pernambuco na cidade do Recife.

A análise da performance dos atletas paraolímpicos de natação é uma tarefa muito complexa. A complexidade advém da grande gama de deficiências apresentadas pelos nadadores. Entenda-se aqui não deficiência técnica, mas física. Os parâmetros de comparação do desenvolvimento técnico apresentado por esses atletas ainda continua sendo aqueles descritos na literatura, ou seja, os parâmetros dos competidores olímpicos. Sob esse prisma o atual desenvolvimento técnico da equipe de natação paraolímpica brasileira deixa um pouco a desejar. A ausência de parâmetros relativos de comparação é uma deficiência que devemos superar, a fim de que as análises sejam melhor conduzidas.

Nesse trabalho usamos como parâmetros de comparação a técnica olímpica de performance, não nos esquecendo contudo de considerar as limitações apresentadas pelos atletas.

As filmagens puderam mostrar, de maneira geral, o estado atual de desenvolvimento dos atletas brasileiros, o que passo agora a relatar, sem contudo relacionar observações com observados.

\section{As saídas}

As saídas foram executadas das mais diferentes formas: do bloco de saída com ajuda e sem ajuda, da água com e sem ajuda. Aqueles que não têm movimentos dos membros inferiores e saem de cima (do bloco), apenas caem na água, o mesmo ocorrendo com aqueles que com problema semelhante saem de dentro da água.

Aqueles que apresentam controle dos movimentos nos membros inferiores fazem saídas relativamente boas, porém com deficiências na fase aérea e conseqüentemente na penetração na água. Os erros aparecem de maneira geral na entrada na água, com todo o corpo tocando ao mesmo tempo ou quase ao mesmo tempo na água.

A fase de deslize algumas vezes vem sendo prejudicada pelo início prematuro das batidas de pernas e rotação de braços.

Movimentos indesejáveis na fase aérea (extensão dos ombros) e movimentos desejáveis e necessários que porventura não são executados nessa fase (flexão de joelhos e/ou quadris), assim como posturas inadequadas (flexão dorsal dos tornozelos, cabeça fora de alinhamento, membros superiores afastados etc.) e início prematuro dos movimentos de pernas e braços durante o deslize são plenamente, nesses casos, passíveis de correção.

\section{As viradas}

Para aqueles atletas que não têm controle sobre os membros inferiores as viradas se constituem numa etapa crítica da prova. Para esses atletas a melhoria da velocidade de aproximação deve ser enfatizada para que a reação sobre a borda possa ser bem aproveitada.

São fatores determinantes da performance e não observados na maioria dos atletas:

1) alinhamento do corpo após o abandono da parede;

2) rotação rápida do corpo com flexão nos quadris e joelhos;

3) preparação do posicionamento do corpo para tocar a borda.

\section{Os nados}

Para a atleta deficiente visual seria importante ressaltar o treinamento de alinhamento para que não haja oscilação lateral (de uma raia à outra), aumentando dessa forma o percurso da prova e desacelerando a cada toque na raia. Esse tipo de comportamento pode estar sendo gerado por um erro técnico, ou seja, ultrapassagem da linha média do corpo pelo braço durante a entrada do mesmo na água.

Foram observados diversos comportamentos inadequados que eram gerados por deficiência técnica ou pela própria deficiência apresentada por cada um. 
Muitos atletas mostraram uma quebra no ritmo das braçadas, rotação demasiada de um dos ombros no nado costas, cruzamento da linha média do corpo durante a fase aérea no nado costas, rotação demasiada de todo o corpo no nado costas, tração deficiente na fase principal tanto do nado livre quanto do nado costas. A deficiência na tração dos nados aparece sob várias formas, dentre elas: falta de manutenção da posição adequada das mãos na fase subaquática; membros superiores estendidos na maior parte da tração; no nado peito foi observada a abertura lateral exagerada dos membros superiores; recolhimento precoce dos cotovelos no final da fase de tração.

A baixa de velocidade observada durante a fase subaquática pode ser explicada não por alguma falha técnica, mas sobretudo pelos comprometimentos físicos apresentados por cada um dos atletas.

As oscilações laterais do corpo foram observados em todos aqueles atletas que não apresentavam controle sobre as partes baixas do corpo (abdômen e membros inferiores) e que foram algumas vezes potencializados por deficiências na técnica de tração (nado costas e crawl).

As batidas de pernas apresentaram algumas pequenas deficiências, quais sejam: flexão excessiva dos joelhos na pernada de borboleta e saída da perna da água no nado crawl.

\section{CONCLUSÃO}

A análise técnica das performances no atletismo e na natação mostraram que:

1) os atletas apresentam enorme potencial de progresso técnico em especial os atletas de atletismo;
2) alguns atletas de natação devido aos comprometimentos oriundos da própria deficiência não apresentam, aparentemente, potencial de melhoria técnica, porém há enorme potencial de crescimento no ponto de vista atlético (melhoria de parâmetros fisiológicos);

3) os atletas precisam passar por uma avaliação técnica bianual;

4) métodos e processos de controle de treinamento (parte técnica) precisam ser desenvolvidos para os atletas de modalidades paraolímpicas;

5) padrões de movimento precisam ser descritos e avaliados para cada tipo de deficiência, a fim de que se tenha um padrão de normalidade para futuras comparações.

\section{AGRADECIMENTOS}

- Comitê Paraolímpico Brasileiro (CPB)

- Secretaria Nacional de Esportes

- Rede Cenesp/Unifesp

- Associação Fundo de Incentivo à Psicofarmacologia (Afip)

- Universidade Federal de Uberlândia (UFU)

\section{REFERÊNCIAS}

1. Ballreich R. Grundlagen der biomechanik des sports. Stuttgart: Ferdinand Enke, 1996.

2. Menzel HJ. Conceito de pesquisa e do ensino da biomecânica no esporte. Revista brasileira de ciência e movimento 1997;8:52-8.

3. Winter DA. Biomechanics of human movement. Ontário: John Wiley \& sons, 1979.

4. Baumann W. Métodos de medição e campos de aplicação da biomecânica: estado da arte e perspectivas. VI Congresso Brasileiro de Biomecânica. Brasília, Conferência, 1995.

5. Amadio AC. Fundamentos biomecânicos para a análise do movimento humano. São Paulo, EEFUSP,1996. 